AUTHORS' REPLY We have read with interest the comments of Drs Gianotti and Franconieri. Their observation of a difference in expiratory time during sleep between subjects with intrinsic and extrinsic asthma is interesting but difficult to evaluate. Specifically, it is not clear whether there is a difference in the extent of nocturnal bronchoconstriction between their subjects with intrinsic and extrinsic asthma. As wheeze tends to be associated with prolonged expiration, one would imagine that the longer expiratory time in the patients with intrinsic asthma might have been associated with nocturnal bronchoconstriction. Paragraph 2, however, suggests that the patients with allergic asthma had nocturnal bronchoconstriction but those with intrinsic asthma did not. This requires clarification.

Our own data are not directly comparable with the data of Drs Gianotti and Franconieri, as we did not split our patients into those with intrinsic and extrinsic asthma. Indeed, most had intrinsic asthma.

We believe that our study adequately shows that there are no changes in breathing pattern overnight which are diagnostic of the development of nocturnal bronchoconstriction.

We look forward to reading further details of the study of Drs Gianotti and Franconieri in due course.

AD MORGAN

NJ DOUGLAS

Department of Respiratory Medicine

City Hospital

Edinburgh

\section{Book notice}

Radiologic Atlas of Pulmonary Abnormalities in Children. 2nd edition: D B Singleton, M L Wagner, R V Dutton. (Pp 355; f47.) Sidcup: Saunders (Harcourt Brace and Jovanovich), 1988. ISBN 0-7216-2062-0.

There are eight chapters in this atlas. The first two, dealing with technique and the normal chest radiograph, are both full of useful information. The chapter on surgical or mechanical abnormalities is very broad; there are, however, a few surprising comments. For example, the use of contrast in the upper oesophagus in oesophageal atresia is not routinely accepted; in cystic adenomatoid malformation there is a suggestion that all children require angiography. The section on vascular rings is far from comprehensive -for example, in discussion of the search for an aberrant left pulmonary artery no mention is made of nuclear magnetic resonance of the importance of the lateral view in a barium swallow; angiography is not the best examination for this condition in 1989. The chapter on pulmonary infections, which appropriately occupies over $40 \%$ of the atlas, covers the pathological conditions comprehensively but not the imaging aspects. Gallium is mentioned in the context of pneumocystis infection but not of AIDS. One hundred and thirteen cases are illustrated, yet there are only four computed tomography images. Bronchography, no longer commonly practised, is however included. Tumours, non-infectious primary pulmonary disease, and pulmonary vascular disease are compre- hensively covered, yet no mention is made of MRI, especially $\underset{x}{x}$ as regards the pulmonary veins. The use of DSA angiography for aplasia is questionable. The sporadic recommendation of $\vec{T}$ ultrasound and MRI is disappointing. Radioisotope per- $\bar{C}$ fusion and ventilation scans are not mentioned. There are $\bar{\partial}$ numerous references, but virtually all are fairly old. Nowhere $\overline{\bar{c}}$ in the atlas is the reader given to understand which type of $\vec{\Phi}$ imaging ought to be done for which particular condition and $\varrho$ why. Differential diagnoses are offered. It is surprising that three prominent paediatric radiologists from an excellent $\vec{\theta}$ institution in the United States have failed to include relevant imaging modalities. This volume will be of limited use to both $\overrightarrow{\vec{\omega}}$ paediatricians and radiologists. - IG

\section{Notice}

\section{Dr H M (Bill) Foreman memorial fund}

The trustees of the Dr H M (Bill) Foreman memorial fund invite applications for grants relating to study in respiratory $\vec{b}$ disease. Limited funds are available for registered medical $œ$ practitioners to assist them in travelling to countries other $\bullet$ than their own to study respiratory disease, and also for $\square$ support of clinical research abroad. Intending applicants should write for further details to Dr B H Davies, Sully Hospital, Sully, S Glamorgan CF6 2YA. 